\title{
Custo e lucratividade da produção de sementes de soja enriquecidas com molibdênio ${ }^{1}$
}

\author{
Carina Oliveira e Oliveira ${ }^{2}$, Edson Lazarini ${ }^{2}$, \\ Maria Aparecida Anselmo Tarsitano ${ }^{2}$, Carolina Cipriano Pinto ${ }^{3}$, Marco Eustáquio de Sá ${ }^{2}$
}

\begin{abstract}
Production cost and profitability of soybean seeds enriched with molybdenum

The search for new technologies that reduce costs to produce seeds is very important. This study aimed to analyze the economic viability of producing soybean seeds enriched with molybdenum. The BRS Valiosa RR cultivar was used and four molybdenum doses $\left(0 \mathrm{~g} \mathrm{ha}^{-1}, 200 \mathrm{~g} \mathrm{ha}^{-1}, 400 \mathrm{~g} \mathrm{ha}^{-1}\right.$ and $800 \mathrm{~g} \mathrm{ha}^{-1}$ ) were applied on leaves as it follows: half in the $\mathrm{R}_{3}$ and half in the $\mathrm{R}_{5-4}$ development stages. The total operating cost, gross revenue, operating profit, profitability index, break-even yield and break-even price were estimated. The results showed that the molybdenum foliar application is an economically viable way of producing molybdenum-enriched soybean seeds.
\end{abstract}

KEY-WORDS: Glycine max (L.) Merrill; BRS Valiosa RR cultivar; micronutrient.

\section{INTRODUÇÃO}

A soja [Glycine $\max (\mathrm{L}$.$) Merrill] tem grande$ importância no cenário mundial, tanto no âmbito alimentar quanto econômico. Seus grãos atendem o mercado de produção de farelo, farinhas, óleo, bebidas à base de soja e outros produtos. Segundo dados da Conab (2013), a área plantada com soja é de 27,72 milhões de hectares, apresentando crescimento de $10,7 \%$, em relação à safra anterior.

No Brasil, a produção de soja se torna viável devido ao processo de fixação biológica de nitrogênio, uma vez que a inoculação com Bradyrhizobium possibilita economia anual aproximada de 100-200 $\mathrm{kg} \mathrm{ha}^{-1}$ de $\mathrm{N}$, representando economia aos produtores brasileiros de cerca de US $\$ 3$ bilhões, em fertilizantes nitrogenados (Fagan

\section{RESUMO}

A busca por novas tecnologias que visem a diminuir os custos de produção de sementes é de fundamental importância. O presente estudo objetivou avaliar a viabilidade econômica da produção de sementes de soja enriquecidas com molibdênio. Foram utilizadas a cultivar BRS Valiosa RR e quatro doses de molibdênio ( $0 \mathrm{~g} \mathrm{ha}^{-1}, 200 \mathrm{~g} \mathrm{ha}^{-1}, 400 \mathrm{~g} \mathrm{ha}^{-1}$ e $\left.800 \mathrm{~g} \mathrm{ha}^{-1}\right)$, em duas aplicações via foliar: metade no estádio $R_{3}$ e metade em $\mathrm{R}_{5-4}$. Foram estimados o custo operacional total, receita bruta, lucro operacional, índice de lucratividade, produtividade de equilíbrio e preço de equilíbrio. Concluiu-se que a aplicação foliar de molibdênio é uma forma economicamente viável de produção de sementes de soja enriquecidas com esse micronutriente.

PALAVRAS-CHAVE: Glycine $\max (\mathrm{L}$.) Merrill; cultivar BRS Valiosa RR; micronutriente.

et al. 2007). Esse processo se torna essencial para a produção de soja, pois seus grãos são ricos em proteína e lipídio, e o nitrogênio é imprescindível à síntese de proteína.

Segundo Sfredo \& Oliveira (2010), o molibdênio (Mo) faz parte da nitrogenase sintetizada por bactérias que realizam simbiose com plantas de soja. Assim, a aplicação de Mo pode tornar a fixação biológica de $\mathrm{N}$ mais eficiente. Porém, a disponibilidade de Mo é afetada em solos de $\mathrm{pH}$ ácido, pois esses são ricos em óxidos de ferro e alumínio, que adsorvem os íons de Mo.

Os solos de regiões tropicais são muito intemperizados e, portanto, ricos em $\mathrm{Fe}$ e $\mathrm{Al}$, ocorrendo, com frequência, deficiência de Mo (Sfredo et al. 1997). Devido a essa baixa disponibilidade, faz-se necessário o fornecimento de Mo por outros métodos.

1. Trabalho recebido em jan./2014 e aceito para publicação em jan./2015 (http://dx.doi.org/10.1590/1983-40632015v4527961).

2. Universidade Estadual Paulista "Júlio de Mesquita Filho" (Unesp), Faculdade de Engenharia, Departamento de Fitotecnia, Tecnologia de Alimentos e Socioeconomia, Ilha Solteira, SP, Brasil.E-mails: carina.oliveiras@ymail.com, lazarini@agr.feis.unesp.br, maat@agr.feis.unesp.br, marcosa@agr.feis.unesp.br.

3. Universidade Estadual Paulista "Júlio de Mesquita Filho" (Unesp), Faculdade de Ciências Agrárias e Veterinárias, Departamento de Produção Vegetal, Jaboticabal, SP, Brasil.E-mail: carolinacipriano@ymail.com. 
Segundo Milani et al. (2008), a pulverização foliar de Mo, nos estádios reprodutivos, resulta em produção de sementes com altos teores desse elemento.

A obtenção de sementes com diferentes teores de determinado elemento, em sua composição química, é possível, pois, segundo Guimarães (1999), além dos fatores genéticos, o fornecimento de nutrientes é um prática que afeta a composição química da semente, caso seja realizada em períodos adequados.

Trabalhos como os de Milani et al. (2008) e Nakao et al. (2014) destacam que as sementes colhidas dos experimentos em que se realizava a aplicação de Mo, nos estádios reprodutivos, apresentavam elevado teor de Mo em sua composição. Segundo Campo \& Hungria (2003), para a produção de sementes enriquecidas com molibdênio, devem ser realizadas duas aplicações foliares de $400 \mathrm{~g}$ de Mo, utilizando-se uma fonte solúvel em água, nos estádios $\mathrm{R}_{3}$ (final da floração) e $\mathrm{R}_{5-4}$ (maioria das vagens entre $50 \%$ e $75 \%$ de granação), com intervalo mínimo de 10 dias.

Campo \& Hungria (2003) constataram que plantas de soja oriundas de sementes com elevado teor de molibdênio apresentam maior eficiência no processo de fixação biológica de nitrogênio e maior rendimento. Além disso, esse micronutriente e o cobalto incrementam a germinação e a emergência das sementes em campo (Guerra et al. 2006).

$\mathrm{O}$ crescimento da área cultivada com soja vem acompanhado pelo aumento da demanda por sementes, resultando em gastos significativos, estimados em $12 \%$ do custo total de produção (Agrianual 2014).

A estimativa e a análise dos custos e da lucratividade das atividades, como ferramenta de gestão e auxílio na tomada de decisão, é fundamental para o produtor rural. Assim, este estudo objetivou avaliar a viabilidade econômica da produção de sementes de soja enriquecidas com Mo.

\section{MATERIAL E MÉTODOS}

Os ensaios foram implantados em área experimental $\left(20^{\circ} 21^{\prime} \mathrm{S}, 51^{\circ} 21^{\prime} \mathrm{W}\right.$ e $335 \mathrm{~m}$ de altitude) com Latossolo Vermelho distrófico (LVd) de textura argilosa (Embrapa 2006), cultivado há vários anos com soja e milho, em sistema plantio direto.

A semeadura da soja ocorreu em 06/12/2011, utilizando-se densidade de 16 sementes por metro de sulco, em área sob plantio direto, onde a cultura antecessora era o milho, e a colheita em 18/04/2012. Para o preparo da área, foi realizada dessecação e, posteriormente, utilizou-se triton, para manejar os restos culturais.

A cultura foi irrigada com o auxílio de pivô central e o manejo feito com os seguintes coeficientes da cultura $(\mathrm{Kc})$ : 0,4 no período de estabelecimento da cultura; 0,8 no período vegetativo; 1,10 na época do florescimento; 0,8 no enchimento de grãos; e 0,5 na maturação.

A adubação básica no sulco de semeadura, de acordo com as características químicas do solo, foi de $300 \mathrm{~kg} \mathrm{ha}^{-1}$ da formulação 00-20-20. As sementes foram previamente tratadas com fungicida à base de carboxin + thiram, na concentração de $250 \mathrm{~mL}$ do p.c./ $100 \mathrm{~kg}$ de sementes, e inoculadas com inoculante líquido, seguindo-se as recomendações do fabricante.

O delineamento experimental foi o de blocos ao acaso (uma cultivar e 4 doses de molibdênio), com quatro repetições (Tabela 1). As parcelas foram constituídas por 7 linhas espaçadas em $0,45 \mathrm{~m} \mathrm{e}$ com 50,0 $\mathrm{m}$ de comprimento. Como área útil, foram consideras as 3 linhas centrais de $6,0 \mathrm{~m}$.

A fonte de Mo utilizada apresentava as seguintes características: $15 \%$ de Mo e densidade de $1,32 \mathrm{~g} \mathrm{~L}^{-1}$. A aplicação foi realizada em duas épocas: 29/02/2012 e 14/03/2012.

A solução com molibdênio foi aplicada com pulverizador de barras de $12,0 \mathrm{~m}$, acoplado a um trator, calibrado para aplicação do volume de calda exigido pelo fabricante do produto. O pulverizador estava equipado com bicos do tipo jato leque, espaçados em 0,50 m.

Durante a condução do experimento, foram realizadas as seguintes atividades: 03/01/2012 - aplicação de 40 g i.a. ha-1, imazethapyr $+72 \mathrm{~g}$ i.a. ha ${ }^{-1}$, lactofen +15 g i.a. ha- ${ }^{-1}$, chlorimuron-ethyl; 17/01/2012 - 1,5 L ha $^{-1}$ de glifosato;

Tabela 1. Épocas de aplicação de molibdênio e total aplicado, via foliar, para cada tratamento estudado (Selvíria, MS, 2011/2012).

\begin{tabular}{crcc}
\hline \multirow{2}{*}{ Tratamento } & $\begin{array}{c}\text { Aplicação } \\
\text { em R }_{3}\end{array}$ & $\begin{array}{c}\text { Aplicação } \\
\text { em R }_{5.4}\end{array}$ & $\begin{array}{c}\text { Total } \\
\text { aplicado }\end{array}$ \\
\cline { 2 - 4 } & & $\mathrm{g} \mathrm{ha}^{-1}$ & \\
\hline Testemunha & 0 & 0 & 0 \\
T1 & 100 & 100 & 200 \\
T2 & 200 & 200 & 400 \\
T3 & 400 & 400 & 800 \\
\hline
\end{tabular}


18/01/12 - 90 g i.a. ha ${ }^{-1}$, imidacloprid + beta-ciflutrina + 50 g i.a. ha ${ }^{-1}$, cipermetrina +105 g i.a. ha $^{-1}$, piraclostrobina + epoxiconazol; 20/01/2012 - 700 g i.a. ha-1, endosulfan; 30/01/2012 - 10 g i.a. ha ${ }^{-1}$, clorantraniliprole +84 g i.a. ha ${ }^{-1}$, azoxistrobina + ciproconazol; 15/02/2012 - 50 g i.a. ha ${ }^{-1}$, cipermetrina + 600 g i.a. ha ${ }^{-1}$, paration metílico; 29/02/2012 - $1^{\text {a }}$ aplicação de molibdênio; 04/03/2012 - 66,5 g i.a. ha-1, cipermetrin +25 g i.a. ha-1 ${ }^{-1}$ piraclostrobina + epoxiconazol; 14/03/2012 - 2a aplicação de molibdênio; 15/03/2012 - 84 g i.a. ha ${ }^{-1}$, imidacloprido + beta-ciflutrina $+600 \mathrm{~g}$ i.a. ha ${ }^{-1}$, paration metílico + 50 g i.a. ha ${ }^{-1}$, ciproconazol; 28/03/2012 - 50 g i.a. ha ${ }^{-1}$, cipermetrina $+600 \mathrm{~g}$ i.a. ha $^{-1}$, paration metílico; 18/04/2012 - colheita.

A produtividade das plantas que receberam aplicação de molibdênio via foliar foi calculada pela coleta de todas as plantas da área útil (3 linhas de $6,0 \mathrm{~m}$ ). Essas plantas, após identificação e secagem a sol, foram trilhadas mecanicamente, as sementes obtidas foram pesadas e foi calculada a produtividade de sementes por hectare. Após a pesagem, foi determinada a umidade das sementes, para correção da produtividade a $13 \%$ (base úmida).

Para o cálculo do custo de produção, foi utilizada a estrutura do custo operacional total de produção adotada pelo Instituto de Economia Agrícola (IEA), detalhada em Martin et al. (1998). O custo operacional efetivo (COE) é composto pelas despesas com operações mecanizadas, operações manuais e materiais consumidos. Foram acrescentadas ao COE outras despesas como transporte, limpeza, secagem e estocagem, tendo-se o custo operacional total (COT). Na metodologia do COT, não é considerado o custo de oportunidade do uso dos fatores de produção.

Os custos foram obtidos com base nos seguintes itens: a) operações manuais - foi realizado um levantamento das necessidades de mão de obra nas atividades, durante o ciclo de produção da cultura, relacionando, para cada operação realizada, o número de homens/dia (HD) para executá-la, sendo, em seguida, multiplicado o coeficiente técnico de mão de obra pelo valor médio da região; b) gastos com materiais - obtidos mediante o produto entre a quantidade de materiais usados e os seus respectivos preços de mercado (valor unitário); c) outras despesas - foi considerada a taxa de $5 \%$ do total das despesas com o COE (Miranda et al. 2011, Gitti et al. 2012, Gerlach et al. 2013).
Para determinar a lucratividade dos tratamentos envolvidos, segundo Martin et al. (1998) e Nogueira (2007), foram calculados: a) receita bruta (RB) (em R \$): multiplicação entre a produção $(\mathrm{kg})$ obtida e o preço médio de venda das sementes (em R\$); b) lucro operacional (LO): $\mathrm{LO}=\mathrm{RB}$ - COT; c) índice de lucratividade (IL): $\mathrm{IL}=(\mathrm{LO} / \mathrm{RB}) * 100$, sendo entendido como a proporção da receita bruta que se constitui em recursos disponíveis, após a cobertura do COT da produção; d) preço de equilíbrio: preço equilíbrio $=\mathrm{COT} /$ produção, sendo entendido como o preço mínimo a ser obtido para se cobrir o COT da produção, considerando-se a produtividade média do produtor; e) produtividade de equilíbrio: produtividade equilíbrio $=\mathrm{COT} /$ preço médio de venda, entendida como a produtividade mínima para se cobrir o COT pelo preço pago ao produtor.

Para o custo operacional total (COT), produtividade e receita bruta, foi realizada análise estatística entre os tratamentos, ajustando-se a regressão linear, utilizando-se o programa estatístico Sisvar.

Os preços médios constantes dos insumos e da semente de soja foram levantados no Instituto de Economia Agrícola, no primeiro semestre de 2013, e apresentados em reais (R\$) (IEA 2013). Para os cálculos de lucratividade, o preço recebido pelo produtor, para a venda da semente de soja, foi considerado o mesmo pago por ele pela semente, ou seja, de $R \$ 2,50 \mathrm{~kg}^{-1}$, uma vez que este trabalho objetivou a produção de sementes, e não de grãos.

\section{RESULTADOS E DISCUSSÃO}

Na Tabela 2, encontra-se a estrutura do custo operacional total (COT), para a cultura da soja. O COT considerado é referente ao tratamento que utilizou a menor dose do fertilizante foliar (200 $\left.\mathrm{g} \mathrm{ha}^{-1} \mathrm{de} \mathrm{Mo}\right)$.

Verifica-se que o COT atingiu R \$ 1.863,22 $\mathrm{ha}^{-1}$ (Tabela 2). As despesas com materiais, seguidos das operações mecanizadas, apresentaram os maiores custos, representando $52,96 \%$ e $40,7 \%$ do custo total, respectivamente.

Dentre as despesas com materiais, as mais onerosas foram as dos defensivos, que representaram $42,8 \%$ do COT, seguidas pelas despesas com fertilizantes $(37,43 \%)$. Menegatti \& Barros (2007), analisando o custo de produção de soja convencional e transgênica, observaram que, para ambas, os custos com defensivos e fertilizantes foram os maiores, sendo que, para a soja transgênica, representaram $41 \%$ do custo total. 
Os valores de custos operacionais totais, produtividade e receita bruta de todos os tratamentos estão expostos na Tabela 3.

Para o COT, observa-se que o menor valor ocorreu na testemunha, visto que, para o custo desta, não se realizou a aplicação de molibdênio, reduzindo-se duas pulverizações, em relação aos demais tratamentos. A diferença do COT, para os tratamentos que receberam Mo foliar, está na quantidade do produto. No entanto, as diferenças de custo não são tão grandes, como ocorre na testemunha, em relação aos tratamentos com Mo, pois o custo do produto é baixo: $0,8 \%$ do custo operacional total (Tabela 2).
Vale lembrar que o fertilizante aplicado via foliar trata-se de um micronutriente (Mo) que traz benefícios à cultura da soja. Segundo Malavolta (1994), o Mo é o segundo micronutriente em número de ocorrências de deficiência para essa cultura, sendo a seguinte ordem sugerida pelo autor: $\mathrm{Zn}>\mathrm{Mo}>\mathrm{B}>\mathrm{Cu}=\mathrm{Mn}>\mathrm{Fe}$. A aplicação foliar é o método em que a planta mais aproveita esse micronutriente (Berger et al. 1996).

As produtividades foram aumentando com as doses de molibdênio aplicadas via foliar, com diferença de $680 \mathrm{~kg} \mathrm{ha}^{-1}$ entre a testemunha e a maior dose aplicada (Tabela 3). Nakao et al. (2014) estudaram a aplicação de Mo via foliar, nos estágios reprodutivos, a fim de obterem sementes enriquecidas, e constata-

Tabela 2. Estimativa do custo operacional total obtido com a cultura da soja, em função da aplicação foliar de fertilizante, correspondente ao tratamento com menor dose do fertilizante (200 $\mathrm{g} \mathrm{ha}^{-1} \mathrm{de}$ Mo) (Selvíria, MS, 2011/2012).

\begin{tabular}{|c|c|c|c|c|c|}
\hline Descrição & Especificação & $\mathrm{n}^{\mathrm{o}}$ de vezes & Quantidade & Valor unitário (R\$) & Valor total $(\mathrm{R} \$)$ \\
\hline \multicolumn{6}{|l|}{ A. Operações mecanizadas } \\
\hline Semeadura & HM & 1,00 & 0,40 & 130,00 & 52,00 \\
\hline Pulverização & HM & 9,00 & 0,40 & 81,00 & 291,60 \\
\hline Triton & $\mathrm{HM}$ & 1,00 & 0,70 & 6,25 & 4,38 \\
\hline Colheita & HM & 1,00 & 0,60 & 200,00 & 120,00 \\
\hline Irrigação & $\mathrm{R} \$ \mathrm{~mm}^{-1}$ & 3,00 & 50,00 & 1,50 & 225,00 \\
\hline Pulverização fertilizante foliar & HM & 2,00 & 0,40 & 81,00 & 64,80 \\
\hline Subtotal A & & & & & 757,78 \\
\hline \multicolumn{6}{|l|}{ B. Operações manuais } \\
\hline Inspeção da área & HD & 2,00 & 0,20 & 50,00 & 20,00 \\
\hline Serviço braçal & HD & 1,00 & 0,20 & 50,00 & 10,00 \\
\hline Subtotal B & & & & & 30,00 \\
\hline \multicolumn{6}{|l|}{ C. Materiais } \\
\hline Semente soja & $\mathrm{kg}$ & 1,00 & 65,00 & 2,50 & 162,50 \\
\hline Tratamento semente & $\mathrm{L}$ & 1,00 & 0,16 & 28,40 & 4,63 \\
\hline Fertilizante 00-20-20 & $\mathrm{t}$ & 1,00 & 0,30 & $1.231,30$ & 369,39 \\
\hline Herbicida 1 & $\mathrm{~L}$ & 1,00 & 0,40 & 18,00 & 7,20 \\
\hline Herbicida 2 & $\mathrm{~L}$ & 1,00 & 0,30 & 45,00 & 13,50 \\
\hline Herbicida 3 & $\mathrm{~g}$ & 1,00 & 15,00 & 0,12 & 1,80 \\
\hline Herbicida 4 & $\mathrm{~L}$ & 2,00 & 1,50 & 11,23 & 33,69 \\
\hline Fungicida 1 & $\mathrm{~L}$ & 1,00 & 0,70 & 77,28 & 54,10 \\
\hline Fungicida 2 & $\mathrm{~L}$ & 1,00 & 0,35 & 126,70 & 44,35 \\
\hline Fungicida 3 & $\mathrm{~L}$ & 1,00 & 0,50 & 67,00 & 33,50 \\
\hline Inseticida 1 & $\mathrm{~L}$ & 1,00 & 1,50 & 40,65 & 60,98 \\
\hline Inseticida 2 & $\mathrm{~L}$ & 1,00 & 0,85 & 25,00 & 21,25 \\
\hline Inseticida 3 & $\mathrm{~L}$ & 1,00 & 2,00 & 22,25 & 44,50 \\
\hline Inseticida 4 & $\mathrm{~L}$ & 1,00 & 0,05 & 504,85 & 25,24 \\
\hline Inseticida 5 & $\mathrm{~L}$ & 1,00 & 3,00 & 27,40 & 82,20 \\
\hline Adjuvante & $\mathrm{L}$ & 1,00 & 1,00 & 14,00 & 14,00 \\
\hline Fertilizante via foliar & $\mathrm{L}$ & 2,00 & 0,10 & 70,00 & 14,00 \\
\hline Subtotal C & & & & & 986,82 \\
\hline Custo operacional efetivo (COE) & & & & & $1.774,59$ \\
\hline Outras despesas & & & & & 88,73 \\
\hline Custo operacional total (COT) & & & & & $1.863,32$ \\
\hline
\end{tabular}


ram ganho de produtividade de $486 \mathrm{~kg} \mathrm{ha}^{-1}$, com o uso de $800 \mathrm{~g} \mathrm{ha}^{-1}$, em relação à testemunha.

Como o preço de venda do quilo do produto foi considerado o mesmo para todos os tratamentos, o que variou, para o cálculo da receita bruta, foi a produtividade dos tratamentos. A maior dose de Mo aplicada apresentou receita bruta de R\$1.700,00 a mais do que a testemunha.

A Tabela 4 apresenta os valores de lucro operacional, índice de lucratividade, produtividade de equilíbrio e preço de equilíbrio, em relação aos tratamentos realizados.

O lucro operacional foi positivo, assim como o índice de lucratividade, mostrando que todos os tratamentos foram viáveis economicamente. Porém, ao se realizar a aplicação de molibdênio, nota-se que os tratamentos que receberam Mo foliar apresentaram ganhos mais elevados, em relação à testemunha, chegando a $26,68 \%$ a mais, do T3 para a testemunha.

$\mathrm{O}$ índice de lucratividade demonstra que, a cada R \$ 1,00 gasto com o processo de produção, gerou-se o equivalente a $\mathrm{R} \$ 0,77 ; \mathrm{R} \$ 0,79 ; \mathrm{R} \$ 0,79$; e $\mathrm{R} \$ 0,80$ de renda líquida, respectivamente para a testemunha, T1, T2 e T3.

Pode-se verificar que quanto maior a dose de Mo aplicada via foliar, maior será a produtividade de equilíbrio, pois, para esse cálculo, as variáveis são o preço de venda e o COT de cada tratamento (Tabela 4). Como o preço do quilo da semente foi considerado o mesmo ( $\$$ \$2,50), a variável que muda é o COT, e este foi aumentando (Tabela 3), conforme se aplicaram maiores doses de Mo.

Richetti (2012), estudando a viabilidade econômica na cultura da soja transgênica, obteve produtividade de equilíbrio de $2.448 \mathrm{~kg} \mathrm{ha}^{-1}$. Essa diferença de valores observada entre o trabalho de Richetti (2012) e o presente estudo deve-se ao preço de venda do produto. Enquanto, neste trabalho, o produto final foi vendido como semente, no trabalho de Richetti (2012), o produto foi vendido como grão, que possui valor de mercado inferior. Já o preço de equilíbrio comportou-se de maneira inversa à produtividade de equilíbrio, isso porque ele varia conforme a produtividade de cada tratamento.

Com a diminuição das doses de Mo foliar, menores são as produtividades e, consequentemente, maiores são os preços de equilíbrio (Tabela 3). Assim, enquanto para a testemunha o preço de equilíbrio é de $\mathrm{R} \$ 0,58 \mathrm{~kg}^{-1}$, no tratamento T3, com maior dose de Mo, esse é de $\mathrm{R} \$ 0,51 \mathrm{~kg}^{-1}$. Com isso, no tratamento T3, caso ocorra queda de preço do produto até $\mathrm{R} \$ 0,51 \mathrm{~kg}^{-1}$, o produtor não terá perda de investimento na lavoura.

Neste experimento, como visou-se ao enriquecimento de sementes de soja com molibdênio,

Tabela 3. Custo operacional total (COT), produtividade de sementes e receita bruta obtidos com a cultura da soja, em função da aplicação de fertilizante via foliar (Selvíria, MS, 2011/2012).

\begin{tabular}{ccccc}
\hline \multirow{2}{*}{ Tratamento } & $\begin{array}{c}\text { Operações realizadas } \\
\left(\mathrm{g} \mathrm{ha}^{-1} \mathrm{de} \mathrm{Mo}\right)\end{array}$ & $\begin{array}{c}\text { COT } \\
\left(\mathrm{RS} \mathrm{ha}^{-1}\right)\end{array}$ & $\begin{array}{c}\text { Produtividade } \\
\left(\mathrm{kg} \mathrm{ha}^{-1}\right)\end{array}$ & $\begin{array}{c}\text { Receita bruta } \\
(\mathrm{R} \$)\end{array}$ \\
\hline Testemunha & 0 & $1.780,58$ & 3.070 & $7.675,00$ \\
T1 & 200 & $1.863,32$ & 3.530 & $8.825,00$ \\
T2 & 400 & $1.878,02$ & $8.950,00$ \\
T3 & 800 & $1.907,42$ & 3.580 & $9.375,00$ \\
\hline & & Resultados do teste F da regressão linear & 3.750 & 0,002 \\
& p-valor & 0,000 & 0,002 & 0,78 \\
\hline
\end{tabular}

Tabela 4. Lucro operacional (LO) por hectare, índice de lucratividade (IL), produtividade de equilíbrio e preço de equilíbrio obtidos com a cultura da soja, em função da aplicação de fertilizante via foliar (Selvíria, MS, 2011/2012).

\begin{tabular}{cccccc}
\hline \multirow{2}{*}{ Tratamento } & Operações realizadas & $\mathrm{LO}$ & $\mathrm{IL}$ & Produtividade de equilíbrio & Preço de equilíbrio \\
\cline { 2 - 5 } & $\mathrm{g} \mathrm{ha}^{-1} \mathrm{de} \mathrm{Mo}$ & $\mathrm{R} \$$ & $\%$ & $\mathrm{~kg} \mathrm{ha}$ & $\mathrm{R} \mathrm{kg}^{-1}$ \\
\hline Testemunha & 0 & $5.894,42$ & 76,80 & 712,23 & 0,58 \\
T1 & 200 & $6.961,68$ & 78,89 & 745,33 & 0,53 \\
T2 & 400 & $7.071,98$ & 79,02 & 751,21 & 0,52 \\
T3 & 800 & $7.467,58$ & 79,65 & 762,97 & 0,51 \\
\hline
\end{tabular}


foi determinado o teor de Mo nas sementes colhidas. As sementes dos tratamentos com 200-800 $\mathrm{g} \mathrm{ha}^{-1} \mathrm{de}$ Mo apresentaram teores de 34,84-58,05 $\mathrm{mg} \mathrm{kg}^{-1}$ de Mo, respectivamente. Esse resultado mostra que, se o produtor de sementes realizar a aplicação de Mo nos estádios reprodutivos, obterá sementes enriquecidas com esse elemento sem ter grandes gastos, pois, como observado, o gasto com Mo foi de $0,8 \%$, e a pulverização para a aplicação deste de $3,5 \%$ do COT (Tabela 2). O produtor poderá compensar esse gasto agregando valor ao seu produto final.

$\mathrm{O}$ produtor que comprar sementes enriquecidas com Mo pagará um pouco a mais. No entanto, não necessitará utilizar esse micronutriente junto ao tratamento das sementes, pois as mesmas já terão quantidades adequadas para suprir a demanda, em solos com deficiência desse elemento. Vale, ainda, ressaltar que o uso de Mo e Co na forma salina, junto ao tratamento de sementes, afeta a sobrevivência de bactérias nitrificantes, a nodulação e a eficiência da fixação de $\mathrm{N}_{2}$, pelo efeito osmótico causado pela utilização de sais como fontes desses elementos (Campo et al. 1999).

\section{CONCLUSÃO}

A dose de $800 \mathrm{~g} \mathrm{ha}^{-1}$ de molibdênio (maior dose) aplicada a sementes de soja aumenta o lucro operacional da produção das sementes em 26,69 \%, em relação à testemunha.

\section{AGRADECIMENTOS}

À Fundação de Amparo à Pesquisa do Estado de São Paulo (FAPESP), pela bolsa de estudo.

\section{REFERÊNCIAS}

AGRIANUAL: anuário da agricultura brasileira. São Paulo: FNP, 2014.

BERGER, P. G.; VIEIRA, C.; ARAÚJO, G. A. A. Efeitos de doses e épocas de aplicação de molibdênio sobre a cultura do feijão. Pesquisa Agropecuária Brasileira, Brasília, DF, v. 31, n. 7, p. 473-480, 1996.

CAMPO, R. J.; ALBINO, U. B.; HUNGRIA, M. Métodos de aplicação de micronutrientes na nodulação e na fixação de $\mathrm{N}_{2}$ em soja. Londrina: Embrapa Soja, 1999.

CAMPO, R. J.; HUNGRIA, M. Enriquecimento de sementes de soja com molibdênio como fator de aumento da eficiência da fixação biológica do nitrogênio e do rendimento da soja. Londrina: Embrapa Soja, 2003.

COMPANHIA NACIONAL DE ABASTECIMENTO (Conab). Acompanhamento da safra brasileira de grãos 2012/2013: décimo primeiro levantamento - agosto/2013. Brasília, DF: Conab, 2013.

EMPRESA BRASILEIRA DE PESQUISA AGROPECUÁRIA (Embrapa). Centro Nacional de Pesquisa de Solos. Sistema brasileiro de classificação de solos. 2. ed. Brasília, DF: Embrapa-SPI, 2006.

FAGAN, E. B. et al. Fisiologia da fixação biológica de nitrogênio em soja: revisão. Revista da Faculdade de Zootecnia, Veterinária e Agronomia, Uruguaiana, v. 14, n. 1, p. 89-106, 2007.

GERLACH, G. A. X. et al. Análise econômica da produção de feijão em função de doses de nitrogênio e coberturas vegetais. Pesquisa Agropecuária Tropical, Goiânia, v. 43, n. 1, p. 42-49, 2013.

GITTI, D. C. et al. Influence of nitrogen fertilization and green manure on the economic feasibility of no-tilled wheat in the Cerrado. Revista Ceres, Viçosa, v. 59, n. 2, p. 246-253, 2012.

GUERRA, C. A. et al. Soybean seed physiological quality in function of phosphorus, molybdenum and cobalt fertilization. Acta Scientarum Agronomy, Maringá, v. 28, n. 1, p. 91-97, 2006.

GUIMARÃES, R. M. Fisiologia de sementes. Lavras: UFLa, 1999.

INSTITUTO DE ECONOMIAAGRÍCOLA(IEA). Preços médios mensais pagos pela agricultura. 2013. Disponível em: <http://www.iea.sp.gov.br/out/index.php>. Acesso em: 15 dez. 2013.

MALAVOLTA, E. Fertilizantes e seu impacto ambiental: micronutrientes e metais pesados, mitos, mistificação e fatos. São Paulo: Produquímica, 1994.

MARTIN, N. B. et al. Sistema integrado de custos agropecuários “Custragri”. Informações Econômicas, São Paulo, v. 28, n. 1, p. 7-28, 1998.

MILANI, G. L. et al. Nodulação e desenvolvimento de plantas oriundas de sementes de soja com altos teores de molibdênio. Revista Brasileira de Sementes, Londrina, v. 30, n. 2, p. 19-27, 2008.

MENEGATTI, A. L. A.; BARROS, A. L. M. Análise comparativa dos custos de produção entre soja transgênica e convencional: um estudo de caso para o Estado do Mato Grosso do Sul. Revista de Economia e Sociologia Rural, Brasília, DF, v. 45, n. 1, p. 163-183, 2007.

MIRANDA, L. P. M. et al. Estimativa do custo para implantação de Astronium fraxinifolium em Latossolo 
degradado utilizando-se adubos verdes e lodo de esgoto. Pesquisa Agropecuária Tropical, Goiânia, v. 41, n. 4, p. 475-480, 2011.

NAKAO, A. H. et al. Aplicação foliar de molibdênio em soja: efeitos na produtividade e qualidade fisiológica da semente. Enciclopédia Bioesfera, Goiânia, v. 10, n. 18, p. 343-352, 2014.

NOGUEIRA, M. P. Gestão de custos e avaliação de resultados. 2. ed. Bebedouro: Scot Consultoria, 2007.
RICHETTI, A. Viabilidade econômica da cultura da soja na safra 2012/2013, em Mato Grosso do Sul. Dourados: Embrapa, 2012. (Comunicado técnico, 177).

SFREDO, G. J. et al. Eficácia de produtos contendo micronutrientes, aplicados via semente, sobre produtividade e teores de proteína da soja. Revista Brasileira de Ciência do Solo, Viçosa, v. 21, n. 1, p. 41-45, 1997.

SFREDO, G. J.; OLIVEIRA, M. C. N. Soja: molibdênio e cobalto. Londrina: Embrapa Soja, 2010. (Documentos, 322 ). 